Meta

Journal des tradlucteurs

Translators' Journal

\title{
Les services de recherche informatisés de l'ICIST
}

\section{Robert Serré}

Volume 25, numéro 1, mars 1980

La documentation

URI : https://id.erudit.org/iderudit/004098ar

DOI : https://doi.org/10.7202/004098ar

Aller au sommaire du numéro

\section{Éditeur(s)}

Les Presses de l'Université de Montréal

ISSN

0026-0452 (imprimé)

1492-1421 (numérique)

Découvrir la revue

Citer cet article

Serré, R. (1980). Les services de recherche informatisés de l'ICIST. Meta, 25(1),

178-181. https://doi.org/10.7202/004098ar d'utilisation que vous pouvez consulter en ligne.

https://apropos.erudit.org/fr/usagers/politique-dutilisation/ 


\section{Les services de recherche automatisée de l'ICIST}

RoBert SERRE

traducteur,

ICIST

L'Institut canadien de l'information scientifique et technique (ICIST), division du Conseil national de recherches à Ottawa, conserve le fonds documentaire canadien le plus riche en sciences, en techniques et en médecine.

Le Conseil national de recherches du Canada avait huit ans en 1925 lorsqu'une bibliothèque a été établie pour desservir les laboratoires de recherche. $\mathrm{La}$ taille de la collection augmentant de pair avec l'envergure des laboratoires, de plus en plus de chercheurs dans l'ensemble du pays avaient recours aux services de la bibliothèque. Cette situation de fait a été reconnue officiellement en 1966 : en vertu d'une révision de la Loi sur le Conseil national de recherches, celui-ci était chargé d'exploiter la Bibliothèque scientifique nationale (BSN) au service du Canada. La BSN est devenue l'Institut canadien de l'information scientifique et technique en 1974 et depuis cette date l'ICIST occupe un imposant édifice dans le campus scientifique du CNRC, chemin de Montréal à Ottawa.

Etant donné l'accroissement exponentiel de la masse documentaire mondiale, surtout depuis la Seconde Guerre mondiale, l'acheminement de l'information scientifique et technique se fonde de plus en plus sur des méthodes de stockage, de recherche et de diffusion automatisées. L'Institut a vite compris l'importance de ces méthodes et est devenu le chaînon central d'un vaste réseau documentaire canadien.

Activité relativement récente, la recherche documentaire automatisée a déjà atteint un degré de complexité et de perfectionnement très poussé. Le Canada, bien placé pour profiter des techniques les plus avancées, a mis au point des services bien adaptés à son immense étendue et à ses besoins linguistiques.

Le présent exposé se limitera aux principaux volets de ces services, les exemples étant tirés de cas concrets mettant en jeu un traducteur ou un terminologue.

Une collection scientifique et technique comme celle de l'ICIST est formée à $80 \%$ environ de revues et de publications en série. Les monographies, beaucoup moins nombreuses, sont aussi moins à jour. Par contre, les ouvrages de référence gardent un grand intérêt et les comptes rendus de conférences sont en pleine expansion. 
Le catalogue sur fiches d'une bibliothèque peut bien suffire à cerner les monographies traitant d'un domaine particulier, grâce aux notices AUTEUR, TITRE et SUJET. Par contre, des centaines de milliers d'articles sont publiés chaque année et un chercheur ne saurait identifier aisément ceux qui l'intéressent sans avoir recours aux bulletins de résumés et d'index, ou encore aux fichiers automatisés qui correspondent à la version imprimée des principaux bulletins.

La collection de l'ICIST compte quelque 1460 bulletins de résumés et d'index imprimés, dans tous les domaines scientifiques, techniques et médicaux, mais seuls les plus importants ont donné lieu à des fichiers automatisés. Par contre, ceux-ci sont très riches et couvrent les principaux domaines de la recherche, offrant l'ensemble ou une partie des points d'accès suivants : le nom des auteurs, l'organisme employeur des auteurs, les mots clés du titre des articles et les descripteurs ajoutés durant l'indexation, le titre codé des revues, des codes thématiques, des codes de langue, des codes de genre de document (brevet, rapport technique, thèse, etc.), l'année de publication et ainsi de suite. Cette flexibilité et une grande rapidité font de l'ordinateur un outil de recherche documentaire très précieux.

L'ICIST reçoit bon nombre des grands fichiers documentaires automatisés, sous forme de bandes magnétiques dont les données sont transformées en fonction des services bien précis offerts par l'ICIST aux chercheurs canadiens.

C'est en 1969 que l'Institut lançait son premier service documentaire automatisé, appelé CAN/SDI (Canadian Selective Dissemination of Information ou diffusion sélective de l'information au Canada). Il s'agit d'un programme d'information permanente qui signale régulièrement aux abonnés les dernières parutions dans leurs domaines d'intérêt. Cinq centres CAN/SDI traitent actuellement plus de 3000 profils documentaires sur 18 banques de données bibliographiques. Un profil est une description, par l'abonné, du genre de document qui l'intéresse. Un bon profil doit permettre de repérer le plus grand nombre d'articles pertinents et le plus petit nombre d'articles inutiles. Il convient donc d'y inscrire les mots clés qui décrivent le mieux le domaine d'intérêt, y compris les synonymes et les abréviations et sigles, le nom des auteurs et des établissements particulièrement actifs, la ou les langues jugées utiles. On peut aussi exclure explicitement des documents sans intérêt, comme les articles d'une revue qu'on reçoit déjà. Les premiers résultats permettent de raffiner le profil qui, avec le temps, devient un outil de recherche vraiment personnalisé, qu'on peut d'ailleurs modifier n'importe quand.

Un profil CAN/SDI pourrait très bien venir en aide à un terminologue qui aurait mené une recherche thématique de plusieurs mois sur les matières plastiques par exemple. Pour tenir le fichier à jour, il pourrait demander qu'on lui signale les documents, dans les langues qui l'intéressent, susceptibles de recenser les innovations dans ce domaine en pleine croissance (articles, conférences, brevets). Pour bien rédiger son profil et choisir les fichiers documentaires appropriés (il y en a 18), il pourrait consulter le Manuel de rédaction des profils $C A N / S D I$, publié par l'ICIST, et s'adresser à un rédacteur de recherches qui réviserait le profil, ferait des suggestions et assurerait l'entrée en ordinateur. Le terminologue 
recevrait alors chaque mois, chaque quinzaine, ou chaque semaine selon les fichiers retenus, une bibliographie individuelle qui simplifierait grandement les travaux de mise à jour du fichier terminologique.

Le service CAN/SDI répond à un besoin d'information courante. Il existe aussi un besoin, tout aussi important, d'information rétrospective. L'Institut a donc mis sur pied, à compter de 1973, le service CAN/OLE (Canadian On-Line Enquiry ou interrogation en direct au Canada). Le chercheur s'assoit à un terminal muni d'un clavier et souvent d'un écran et d'une imprimante. Il s'agit de dialoguer directement avec l'ordinateur (d'où l'expression « en direct ») en interrogeant des fichiers qui englobent les références bibliographiques de plusieurs années (d'où l'expression « recherche rétrospective»). Le service CAN/OLE est en pleine expansion : plus de 220 centres canadiens mettant en jeu quelque 475 terminaux permettent actuellement aux chercheurs de consulter en direct sept millions et demi de références bibliographiques regroupées dans douze fichiers. Cette envergure et la puissance du nouvel ordinateur du CNRC font de CAN/OLE, dont la version 3 vient d'être lancée (novembre 1979), un outil très perfectionné. Le tarif, qui varie selon le fichier consulté, est essentiellement fonction du temps d'utilisation. Un nouveau Manuel de l'utilisateur CAN/OLE décrit l'ensemble remanié des instructions qui permettent de dialoguer avec l'ordinateur. De plus, l'ICIST assure la formation du personnel des centres CAN/OLE : un chercheur exercé peut repérer des renseignements bibliographiques très variés avec une rapidité surprenante. Depuis quelque temps, il est même possible de commander directement au terminal les documents jugés particulièrement pertinents. Ceux-ci sont acheminés par le service de prêts interbibliothèques et de reprographie de l'ICIST aux tarifs usuels.

Prenons l'exemple d'un traducteur qui travaille dans un organisme qui a signé un contrat de centre CAN/OLE. Il doit traduire, de l'anglais vers le français, un exposé sur les laveurs de gaz dans l'industrie. Il trouve quelques sources secondaires (encyclopédies, dictionnaires), mais pour se sentir plus à l'aise dans ce domaine qu'il connaît moins bien, il va demander au responsable du centre CAN/OLE d'interroger pour lui le fichier CHEMICAL ABSTRACTS et de repérer des documents en français sur les laveurs de gaz. En moins de cinq minutes, l'ordinateur affiche sur l'écran neuf références bibliographiques, toutes pertinentes. Trois d'entre elles renvoient à des articles publiés dans la revue $L a$ Technique moderne, qu'il peut consulter sur place. Il commande directement les six autres documents, dont quatre ont été publiés dans des revues, les deux derniers étant tirés d'une conférence. Le lendemain, l'ICIST lui fait parvenir une photocopie des quatre articles de revue et lui apprend que les actes de la conférence viennent tout juste d'être catalogués et qu'un exemplaire en double lui sera prêté pour deux semaines.

Dans une autre situation, un terminologue a entrepris une recherche thématique sur la microscopie. Il a établi une riche documentation en anglais, qu'il juge suffisante. Du côté français, il est moins satisfait. Plutôt que de passer un temps précieux à dépouiller des bulletins de résumés, il décide de s'adresser à l'ICIST, qui offre un « service de recherche documentaire unifié ». Le prix de base de $\$ 30$ 
lui donne droit à une recherche bibliographique dans un choix de fichiers automatisés et de bulletins imprimés. Le fichier CHEMICAL ABSTRACTS l'intéresse tout particulièrement puisque l'index langue lui permet d'identifier aisément les documents de langue française, tandis que l'index de codes thématiques lui permet de limiter les documents repérés aux seuls exposés de synthèse, qu'il juge suffisants. Il reçoit sa bibliographie par la poste, sous forme de feuillet qui se détache en fiches individuelles. Ces fiches contiennent tous les renseignements requis pour commander de l'ICIST les documents qu'il ne réussit pas à trouver plus près de lui.

Depuis plusieurs années, l'ICIST accorde également une attention toute particulière aux sciences de la santé. En vertu d'une entente conclue avec la National Library of Medicine (NLM) des Etats-Unis, l'ICIST coordonne un réseau de 74 centres canadiens qui peuvent interroger, en direct, les divers fichiers de la NLM qui constituent le service MEDLINE (recherche documentaire biomédicale automatisée). Les recherches MEDLINE doivent passer par un vocabulaire autorisé ou thésaurus appelé MeSH ( $M E$ dical Subject Headings). Beaucoup plus complexe que CAN/SDI ou CAN/OLE, MEDLINE exige une formation plus poussée ; par contre, c'est un outil de recherche très raffiné et très flexible.

L'ICIST regroupe de nombreuses autres ressources documentaires susceptibles d'être utiles aux traducteurs et aux terminologues, dont quelques-unes sont automatisées : le fichier UNION, version mécanisée d'un catalogue collectif, préparé par l'KCIST, qui donne l'état de près de 48000 revues scientifiques, techniques et médicales conservées dans quelque 250 bibliothèques canadiennes ; le fichier IEC, version mécanisée d'un répertoire, publié par l'ICIST, qui signale quelque 76700 programmes de recherche universitaire subventionnés par le gouvernement fédéral depuis 1971.

Tout cela semble bien éloigné de l'idée traditionnelle qu'on se fait d'une bibliothèque. Les ressources documentaires de l'ICIST annoncent l'avenir ; elles permettent dès maintenant ce dont on ne voudra plus se passer dans quelques années. La recherche documentaire automatisée est une activité toute jeune qui est en train de transformer notre monde profondément, y compris l'exercice de la traduction et de la terminologie. L'écrit et l'imprimé sont au seuil d'une ère nouvelle qui, si on s'y prépare bien, rendra encore plus fascinant le métier de ceux qui aident les hommes à communiquer entre eux. 